\title{
Geometrically nonlinear free and forced vibrations analysis of clamped-clamped functionally graded beams with multi- cracks
}

\author{
Mohcine Chajdi ${ }^{1, *}$, Ahmed Adri ${ }^{2}$, Khalid El bikri ${ }^{1}$, and Rhali Benamar ${ }^{3}$ \\ ${ }^{1}$ Mohammed V University in Rabat, ENSET - Rabat, MSSM, B.P.6207, Rabat Institute, Rabat, Mo- \\ rocco \\ ${ }^{2}$ Hassan II University of Casablanca, EST - CASABLANCA, LMPGI, B.P.8012 Oasis, Casablanca, \\ Morocco \\ ${ }^{3}$ Mohammed V University in Rabat, EMI - Rabat, LERSIM, B.P.765 Agdal, Rabat, Morocco
}

\begin{abstract}
Geometrically nonlinear free and forced vibrations of clampedclamped Functionally Graded beams with multi-cracks, located at different positions, based on the equivalent rotational spring model of crack and the transfer matrix method for beams is investigated. The FG beam properties are supposed to vary continuously through the thickness direction. The theoretical model is based on the Euler-Bernoulli beam theory and the Von Karman geometrical nonlinearity assumptions. A homogenization procedure, taking into account the presence of the crack, is developed to reduce the problem examined to that of an equivalent isotropic homogeneous multi-cracked beam. Upon assuming harmonic motion, the discretized expressions for the total strain and kinetic energies of the beam are derived, and through application of Hamilton's principle and spectral analysis, the problem is reduced to a nonlinear algebraic system solved using an approximate explicit method developed previously (second formulation) to obtain numerically the FG multi-cracked beam nonlinear fundamental mode and the corresponding backbone curves for a wide range of vibration amplitudes. The numerical results presented show the effect of the number of cracks, the crack depths and locations, and the volume fraction on the beam nonlinear dynamic response.
\end{abstract}

\section{Introduction}

In recent years, functionally graded materials (FGM's) have been regarded as one of important developments in new advanced composite materials considered as a possible solution to the problems and limitations of conventional composites. Also, their multiple advantages, such as the good thermal resistance and the absence of delamination problems, often encountered with classical laminated structures, make them more attractive. Numerous methods based on analytical, semi analytical and numerical techniques, available in the open literature, address the nonlinear problem of large vibration amplitudes in both the free and forced regimes. The case of FGM cracked beams have attracted increasing research efforts. The present work aims to investigate the geometrically nonlinear free and forced vibrations of

\footnotetext{
*e-mail: chajdimohcine@gmail.com
} 
clamped-clamped Functionally Graded beams with multi-cracks, located at different positions, based on the Euler-Bernoulli beam theory and the Von Karman geometrical nonlinearity assumptions. A homogenization procedure was used in [1, 2] to reduce the problem under consideration to that of an equivalent isotropic homogeneous multi-cracked beam. The closed-form solutions and transfer matrix method used previously in [3] are employed and the resulting equation is solved iteratively by the Newton Raphson method. The nonlinear case is then examined, by expanding the nonlinear multi-cracked beam transverse displacement function as a series of the linear modes calculated before. The improved model developed in [4] is used to obtain the basic function contribution coefficients to the displacement response function of the multi-cracked beam and the corresponding backbone curves giving the non-linear amplitude-frequency dependence. Comprehensive numerical results are provided to study the effects of the material property gradient, the crack depths and locations, and the number of cracks on the nonlinear free and forced dynamic behaviour of cracked FGM beams.

\section{Problem formulation}

Consider a multi-cracked FG Euler-Bernoulli beam subjected to a harmonic excitation force, having the geometrical characteristics shown in Fig. 1. In the context of the present study, the material properties are assumed to vary according to a continuous exponential function along the depth of beam as follows:

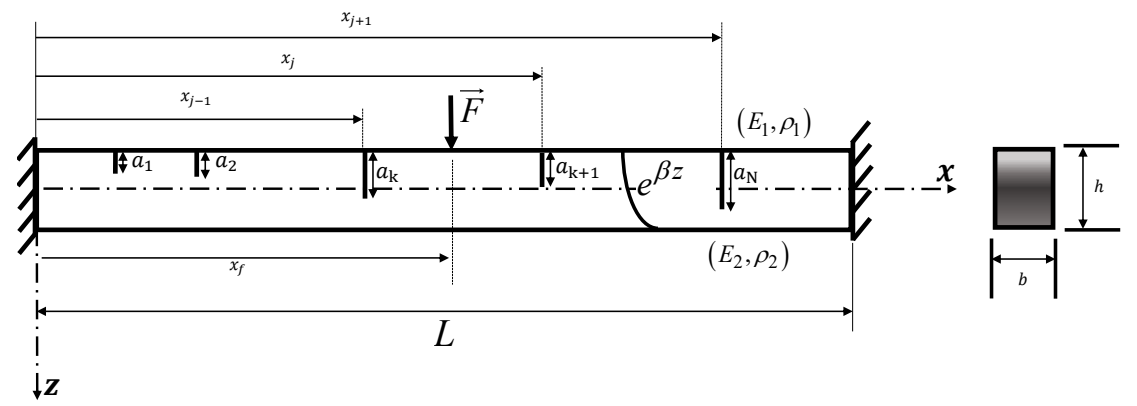

Figure 1. Physical model of the multi-cracked FG beam

$$
\begin{aligned}
& \mathbf{E}(z)=\mathrm{E}_{1} \sqrt{k} \mathrm{e}^{\frac{z}{h} \ln (k)} \\
& \rho(z)=\rho_{1} \sqrt{k} \mathrm{e}^{\frac{z}{h} \ln (k)}
\end{aligned}
$$

The material properties are characterised by the indices $k=E_{2} / E_{1}$ and $\beta=\ln (k / h)$, Note that no variation in the Poisson's ratio $v$ is considered since its influence on the stress intensity factors (SIF) is quite limited [5]. The present formulation, based on the neutral surface approach, is implemented for the forced vibration problem by distinguishing between the beam mid-plan and its neutral surface defined by [6].

$$
\delta=\frac{\int_{-\frac{h}{2}}^{\frac{h}{2}} z \mathbf{E}(z) d z}{\int_{-\frac{h}{2}}^{\frac{h}{2}} \mathbf{E}(z) d z}
$$


It is assumed that the crack remains always open and perpendicular to the beam surface. Based on the rotational spring model, the bending stiffness $K_{\tau}$ of the cracked section is related to the flexibility $C$ by:

$$
C=\int_{0}^{\alpha} \frac{72 \pi\left(1-v^{2}\right) \alpha \mathbf{F}^{2}(\alpha)}{\mathbf{E}(a) h^{2}} d \alpha=\frac{1}{K_{\tau}} \quad \text { with } \quad \mathbf{E}(a)=E_{l} \sqrt{k} e^{\left(-\frac{1}{2}-\frac{\delta}{h}+\alpha\right) \ln (k)}
$$

Where $\alpha=a / h$ is the crack depth. $\mathbf{F}(\alpha)$ is called the crack correction function and $\mathbf{E}(a)$ is the effective elastic modulus at the crack tip.

\section{1 linear vibration analysis}

For the linear vibration analysis, neglecting the nonlinear terms, the transverse and axial displacements in the $j^{\text {th }}$ span $x_{j-1} \leq x \leq x_{j}$ for $j=1,2, \ldots, N+1$ can be expressed as:

$$
\begin{gathered}
\mathbf{w}_{i j}(x)=\mathbf{A}_{j} \cosh \left(\beta_{i} L\left(x-x_{j-1}\right)\right)+\mathbf{B}_{j} \sinh \left(\beta_{i} L\left(x-x_{j-1}\right)\right)+\mathbf{C}_{j} \cos \left(\beta_{i} L\left(x-x_{j-1}\right)\right) \\
+\mathbf{D}_{j} \sin \left(\beta_{i} L\left(x-x_{j-1}\right)\right) \\
\mathbf{u}_{i j}(x)=\frac{B_{11}}{A_{11}}\left[\frac{\partial \mathbf{w}_{i j}(x)}{\partial x}\right]+\mathbf{E}_{j}\left(x-x_{j-1}\right)+\mathbf{F}_{j}
\end{gathered}
$$

The constants $\left(\mathbf{A}_{j}, \mathbf{B}_{j}, \mathbf{C}_{j}, \mathbf{D}_{j}, \mathbf{E}_{j}\right.$ and $\left.\mathbf{F}_{j}\right)$ are determined by the imposed general conditions at the beam ends, continuity and compatibility conditions at the crack location [7]. By using the transfer matrix method, the six constants in the first segment can be mapped into those of the last segment, reducing the total number of independent constants to six.

$$
\left\{\begin{array}{c}
A_{N+1} \\
B_{N+1} \\
C_{N+1} \\
D_{N+1} \\
E_{N+1} \\
F_{N+1}
\end{array}\right\}=\mathbf{T}_{N} \times \ldots \times \mathbf{T}_{2} \times \mathbf{T}_{1}\left\{\begin{array}{c}
A_{1} \\
B_{1} \\
C_{1} \\
D_{1} \\
E_{1} \\
F_{1}
\end{array}\right\}
$$

Where $\mathbf{T}_{j}$ is the $6 \times 6$ transfer matrix which depends on the values of $\beta_{i}$, and consequently on the eigenvalue $\omega_{i}$.

\subsection{Nonlinear vibration analysis}

Using the Euler-Bernoulli theory displacement fields as mentioned in [8] and assuming that the FG cracked beam exhibits large vibration amplitudes, the Von-Karman nonlinear straindisplacement and the elastic plane stress-strain relationships are given by :

$$
\varepsilon_{x}=\frac{\partial \mathbf{u}}{\partial x}+\frac{1}{2}\left(\frac{\partial \mathbf{w}}{\partial x}\right)^{2}-z \frac{\partial^{2} \mathbf{w}}{\partial x^{2}}, \quad \sigma_{x}=\frac{\mathbf{E}(z)}{1-v^{2}} \varepsilon_{x}
$$

The axial force and the bending moment are then obtained as:

$$
\begin{gathered}
\mathbf{N}_{x}=\int_{A} \sigma_{x} d A=\mathbf{A}_{11}\left[\frac{\partial \mathbf{u}}{\partial x}+\frac{1}{2}\left(\frac{\partial \mathbf{w}}{\partial x}\right)^{2}\right]-\mathbf{B}_{11} \frac{\partial^{2} \mathbf{w}}{\partial x^{2}} \\
\mathbf{M}_{x}=\int_{A} \sigma_{x} z d A=\mathbf{B}_{11}\left[\frac{\partial \mathbf{u}}{\partial x}+\frac{1}{2}\left(\frac{\partial \mathbf{w}}{\partial x}\right)^{2}\right]-\mathbf{D}_{11} \frac{\partial^{2} \mathbf{w}}{\partial x^{2}}
\end{gathered}
$$


The stiffness component related terms are defined by:

$$
\left(\mathbf{A}_{11}, \mathbf{B}_{11}, \mathbf{D}_{11}\right)=\int_{-\frac{h}{2}+\delta}^{\frac{h}{2}+\delta} \frac{\mathbf{E}(z)}{1-v^{2}}\left(1, z, z^{2}\right) d z
$$

The strain energy $\mathbf{V}$ of the beam is given as:

$$
\mathbf{V}=\frac{1}{2} \int_{0}^{L}\left[\mathbf{N}_{x}\left(\frac{\partial \mathbf{u}}{\partial x}+\frac{1}{2}\left(\frac{\partial \mathbf{w}}{\partial x}\right)^{2}\right)-\mathbf{M}_{x} \frac{\partial^{2} \mathbf{w}}{\partial x^{2}}\right] d x
$$

With Eq. (3) in mind, $\mathbf{B}_{11}$ vanish as the thickness variation in the Young's modulus is the same as in the isotropic case. The axial inertia being neglected, using the static axial equilibrium equation for the beam i.e. $\partial \mathbf{N}_{x} / \partial x=0$, applying the immovable end conditions ( $u=0$, at $x=0$ and $L$ ) and integrating Eq. (9) with respect to $x$, the potential energy $\mathbf{V}$ can be written only in terms of the transverse displacement $\mathbf{w}$ as follows:

$$
\mathbf{V}=\frac{(\mathbf{E S})_{e f f}}{8 L}\left(\int_{0}^{L}\left(\frac{\partial \mathbf{w}}{\partial x}\right)^{2}\right)^{2}+\frac{(\mathbf{E I})_{e f f}}{2} \int_{0}^{L}\left(\frac{\partial^{2} \mathbf{w}}{\partial x^{2}}\right)^{2} d x
$$

Eq. (13) is effective for replacing the FG beam problem with an equivalent isotropic beam, where $(\mathbf{E S})_{e f f}=A_{11}$ and $(\mathbf{E I})_{e f f}=\left(D_{11}-B_{11}^{2} / A_{11}\right)$ are the effective axial and bending stiffness, respectively. The kinetic energy $\mathbf{T}$, the strain energy of the crack $\mathbf{V}_{c}$ and the potential energy associated to the force $\mathbf{V}_{f}$ are given by:

$$
\begin{gathered}
\mathbf{T}=\frac{1}{2} \int_{0}^{L} \int_{-\frac{h}{2}+\delta}^{\frac{h}{2}+\delta} \rho(z)\left(\frac{\partial \mathbf{w}}{\partial t}\right)^{2} d x \\
\mathbf{V}_{c}=\frac{(\mathbf{E I})_{e f f}^{2}}{2 K_{\tau}}\left(\frac{\partial^{2} w}{\partial x^{2}}\right)_{x=x_{N}}^{2} \\
\mathbf{V}_{f}=\int_{A} \mathbf{F}(x, t) \mathbf{w}(x) d x
\end{gathered}
$$

For a general parametric study, the following dimensionless formulation is used

$$
\mathbf{w}(x)=h \mathbf{w}^{*}\left(\frac{x}{L}\right)=h \mathbf{w}^{*}\left(x^{*}\right)
$$

Upon assuming harmonic motion and expanding the transverse displacement in the form of a finite series $\mathbf{w}^{*}\left(x^{*}, t\right)=\mathbf{a}_{i} \mathbf{w}_{i}^{*}\left(x^{*}\right) \sin \left(\omega^{*} t\right)$ in which $\mathbf{w}_{i}^{*}$ are the FG beam linear mode shapes and applying Hamilton's principle, the following set of nonlinear algebraic equations is obtained

$$
2 \mathbf{a}_{i} \mathbf{K}_{i r}^{*}+3 \mathbf{a}_{i} \mathbf{a}_{j} \mathbf{a}_{k} \mathbf{B}_{i j k r}^{*}-2 \omega^{* 2} \mathbf{a}_{i} \mathbf{M}_{i r}^{*}=\mathbf{F}_{i}^{c *} \text { where } \mathbf{F}_{i}^{c *}=\frac{L^{3} F^{c}}{E I h} \mathbf{w}_{i}^{*}\left(x_{f}\right)
$$

Where $\mathbf{K}_{i r}^{*}, \mathbf{B}_{i j k r}^{*}$ and $\mathbf{M}_{i r}^{*}$ stand for the dimensionless classical rigidity tensor, the nonlinear rigidity tensor and the mass tensor, respectively defined in [1].

\section{Numerical results and discussion}

The FG beam under investigation has the following material and physical parameters: the top surface of the FGM $E_{1}=70 G p a, \rho_{1}=2780 \mathrm{Kg} / \mathrm{m}^{3}$ and slenderness ratio $L / h=10$ with 
$h=0.1 \mathrm{~m}$. In order to perform and verify the numerical calculations, the values of the first three modal frequencies with C-C boundary conditions are calculated and compared with the available data in the literature and listed in Table. 1 , after normalization by $\bar{\omega}=\omega / \sqrt{D_{0} / I_{0}}$ where $D_{0}$ and $I_{0}$ are the corresponding values of an isotropic homogeneous beam $k=1$.

The effects of the cracks, for various values of the material index $k$, on the first nonlinear mode shape, the backbone curves and the nonlinear frequency response curve for an FG beam having three cracks equitably distributed are illustrated in Fig. 2, corresponding to $a_{k} / h=0.3$. It can be observed that a beam having a material index $k=0.2$ corresponding to "top surface rich ceramic" is the most sensitive to the presence of cracks. It is also observed in Fig. 2 (b) that the nonlinear frequency ratio is higher in the case of $k=0.2$ as the linear frequency in this case is minimum and lower, compared to the case of an isotropic beam. By applying the single mode assumption used previously in [10], Eq. (18) is presented in Fig. 2 (c) in the neighbourhood of the first nonlinear mode shape corresponding to $F_{i}^{*}=50$, It is evident from the results that the response curve is less affected by the edge crack when the index $k$ increases. Fig. 3 (a) and (b) depict the influence of the number of cracks on

Table 1. Comparison of the first three non-dimensional natural frequencies of intact FG beams

\begin{tabular}{|c|c|c|c|c|c|c|c|c|c|}
\hline \multirow[t]{3}{*}{ Method } & \multicolumn{3}{|c|}{$E_{1} / E_{2}=0.2$} & \multicolumn{3}{|c|}{$E_{1} / E_{2}=1$} & \multicolumn{3}{|c|}{$E_{1} / E_{2}=5$} \\
\hline & \multicolumn{3}{|c|}{ Modes } & \multicolumn{3}{|c|}{ Modes } & \multicolumn{3}{|c|}{ Modes } \\
\hline & 1 & 2 & 3 & 1 & 2 & 3 & 1 & 2 & 3 \\
\hline Present & 21.02 & 57.94 & 113.59 & 22.37 & 61.67 & 120.90 & 21.02 & 57.94 & 113.59 \\
\hline [8] & 21.02 & 57.94 & 113.59 & 22.37 & 61.67 & 120.90 & 21.02 & 57.94 & 113.59 \\
\hline [9] & 21.02 & 57.94 & 113.59 & 22.37 & 61.67 & 120.90 & 21.02 & 57.94 & 113.59 \\
\hline
\end{tabular}
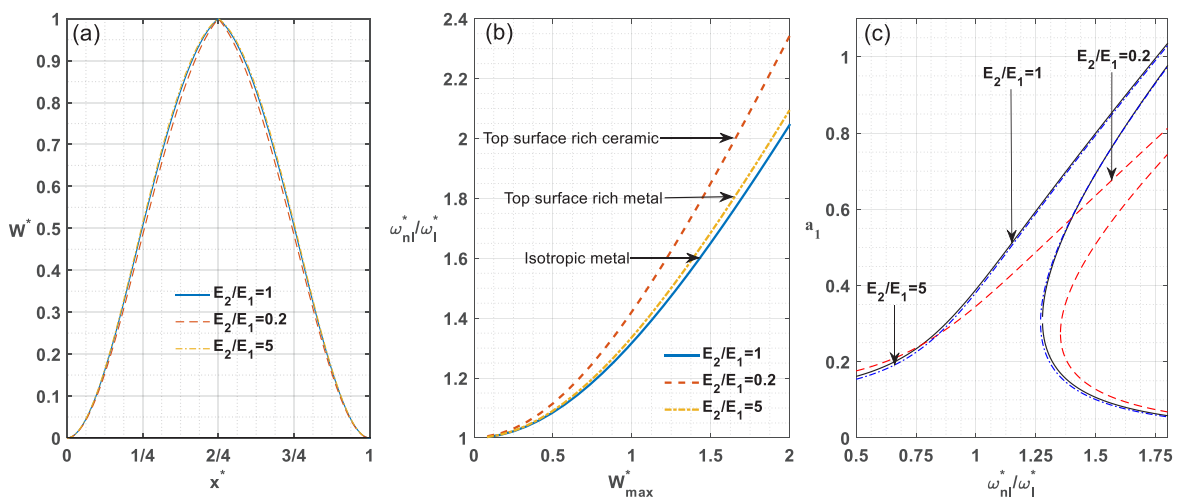

Figure 2. The normalized first non-linear mode shape (a), the backbone curves (b) and the response curves (c) of C-C cracked beam with three open edge cracks, for various value of index $k$

the frequency response of the FG beam with cracks equitably distributed along the whole beam and equitably distributed inside the left half of the beam respectively corresponding to $a_{k} / h=0.5$. It can be seen that the vibration amplitudes and resonant frequencies are more sensitive to the number of cracks in the case of anti-symmetrical distributions. The effects of the crack depth have been also clearly presented in Fig. 3 (c). It can be concluded that the presence of one deep crack may be more prejudicial than multi-cracks of a smaller depth. 

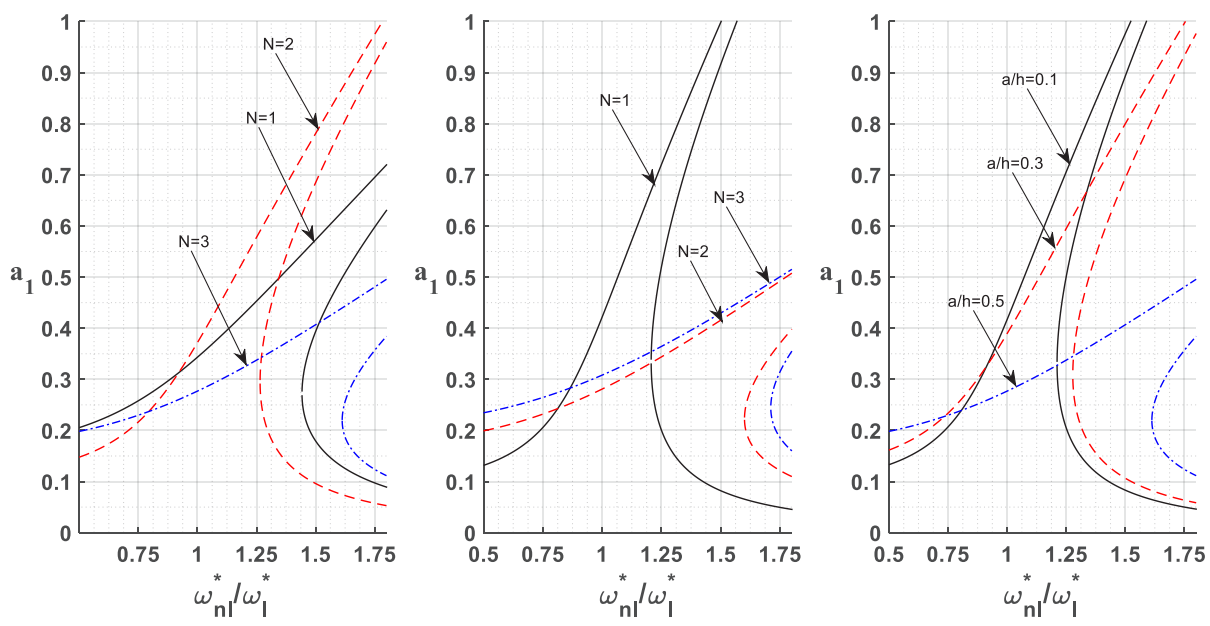

Figure 3. The response curves based on the single mode approach of C-C cracked beam, varying the number of cracks (a)-(b), and the crack depth corresponding to $F_{i}^{*}=50$

\section{Conclusion}

The present study deals with the problem of geometrically nonlinear free and forced vibrations of a clamped-clamped multi-cracked FG beam combining the transfer matrix method with the rotational spring model. A homogenization procedure has been proposed based on the neutral surface approach leading to a simplified formulation of the nonlinear problem which is reduced to that of an isotropic homogeneous beam. A parametric study has been carried out to analyse many effects such as the material composition, the crack depths and locations, on the nonlinear frequencies and mode shapes of FG beams.

\section{References}

[1] M. Chajdi, E. B. Merrimi, and K. El Bikri, Key Eng. Mater. 730, 521-526 (2017)

[2] C. Mohcine, M. E. Bekkaye, and E. B. Khalid, Procedia Eng. 199, 1870-1875 (2017)

[3] A. Adri and R. Benamar, Diagnostyka. 18, no. 2, (2017)

[4] M. El kadiri, R. Benamar, and R. G. White, J. Sound Vib. 249, no. 2, 263-305, (2002)

[5] D. Wei, Y. Liu, and Z. Xiang, Appl. J. Sound Vib. 331, 1686-1700, (2012)

[6] B. Panigrahi and G. Pohit, Appl. Math. Model 57, 248-267, (2018)

[7] I. A. Karnovsky, O. I. Lebed, O. Lebed, and I. Karnovsky, Formulas for Structural Dynamics: Tables, Graphs and Solutions. New York: McGraw-Hill, (2000)

[8] J. Yang and Y. Chen, Compos. Struct. 83, no. 1, 48-60, (2008)

[9] Z. Yu and F. Chu, J. Sound Vib. 325, no. 1, 69-84, (2009)

[10] L. Azrar, R. Benamar, and R. G. White, J. Sound Vib. 224, no. 2, 183-207, (1992) 